\title{
ON A CONSTRUCTION IN BORDISM THEORY
}

\author{
by NIGEL RAY \\ (Received 29th November 1985)
}

\section{Introduction}

In [2], R. Arthan and S. Bullett pose the problem of representing generators of the complex bordism ring $M U_{*}$ by manifolds which are totally normally split; i.e. whose stable normal bundles are split into a sum of complex line bundles. This has recently been solved by Ochanine and Schwartz [8] who use a mixture of $J$-theory and surgery theory to establish several results, including the following.

Theorem 0.1. Each element of $M U_{*}$ may be represented by a manifold which is totally normally split.

Their proof, however, is not explicitly constructive.

It is our aim here to adapt an old construction of Conner and Floyd [4] to give a simple and direct proof of $(0.1)$, which subsumes an explicit choice of generations for $M U_{*}$. Moreover, we attain certain further goals.

Firstly, in answer to a supplementary question of Stong [13], we show that our manifolds have the extra property that they are also totally tangentially split.

Secondly, by considering the symplectic analogue, we exhibit for the first time precise geometrical models for the important elements $\phi_{n} \in M S p_{8 n-3}$ [9] in the symplectic bordism ring.

Additionally, we note that these manifolds, as well as a large family of other $S p$ bordism representatives, are also totally normally and tangentially split.

The key result is in Section 2, where we offer a straightforward procedure for producing generators of $M U_{*}\left(C P^{\infty}\right)$ and $M S p_{*}\left(H P^{\infty}\right)$ over the respective coefficient rings.

Throughout, $E_{*}\left(X_{+}\right)=E_{*} \oplus E_{*}(X)$ stands for the unreduced $E$ homology module of a space $X$. Also, the trivial real, complex or symplectic line bundle over $X$ will be respectively denoted by $\mathbb{R}, \mathbb{C}$ or $\mathbb{H}$, whilst an arbitrary line bundle will be systematically confused with its classifying map.

Special thanks are due to Bob Stong, who spotted an error in an earlier version of this work. It is also a pleasure to acknowledge several enjoyable and influential discussions with Andrew Baker, Shaun Bullett, Elmer Rees and Lionel Schwartz.

\section{Iterated projective bundles}

In this section we describe our basic inductive construction.

To begin, let $B^{0}=*$, admitting the complex line bundle $\beta_{0}=\mathbb{C}$. 
Now suppose given the $2 n-2$ dimensional smooth manifold $B^{n-1}$, carrying line bundles $\beta_{0}, \ldots, \beta_{n-1}$. Then take $B^{n}$ to be the projectification $C P\left(\beta_{n-1} \oplus \mathbb{C}\right)$, with projection $\pi: B^{n} \rightarrow B^{n-1}$ and fibre $C P^{1}$. Each point $\lambda \in B^{n}$ may be considered as a line in $\beta_{n-1} \oplus \mathbb{C}$. Define $\beta_{0}, \ldots, \beta_{n-1}$ over $B^{n}$ by pulling back along $\pi^{*}$, and choose $\beta_{n}$ to be the canonical line bundle whose fibre over $\lambda$ consists of all points in $\lambda$.

Thus $B^{n}$ is a projective variety, and also a smooth $2 n$ dimensional manifold. Moreover, it is diffeomorphic to the 2-sphere bundle $S\left(\beta_{n-1} \oplus \mathbb{R}\right)$ via fibrewise application of the usual identification of $C P^{1}$ with $S^{2}$. In this guise, and for $n>0$, it bounds the disc bundle $D\left(\beta_{n-1} \oplus \mathbb{R}\right)$.

A third description, which we shall not use here, is due to Conner and Floyd [4], and arises from forming the quotient of the cartesian product $\left(S^{3}\right)^{n}$ by a suitable action of the torus $T^{n}$.

We now wish to invest $B^{n}$ with the structure of a weakly almost complex, or $U$ manifold, which is not the obvious projective one. Instead, we take the sphere bundle version, and following Szczarba [16] note that its tangent bundle satisfies

$$
\tau_{n} \oplus \mathbb{R} \cong \pi^{*}\left(\tau_{n-1} \oplus \beta_{n-1} \oplus \mathbb{R}\right)
$$

Since $\tau_{0} \oplus \mathbb{R} \cong \mathbb{R}$, we obtain inductively

$$
\tau_{n} \oplus \mathbb{R} \cong\left(\bigoplus_{i=0}^{n-1} \beta_{i}\right) \oplus \mathbb{R}
$$

This provides an explicit $U$-structure for the stable tangent bundle $\tau_{n}^{S}$, and hence also for the stable normal bundle $v_{n}^{S}$, such that $B^{n}$ is totally tangentially split.

Of course, this $U$-structure may be extended over the corresponding 3-disc bundle.

To study $v_{n}^{S}$ more closely, we recall the well-known splitting principle.

Proposition 1.2. Over $B^{n}$, there is a $U(1)$ bundle $\beta_{n}^{*}$ satisfying

$$
\beta_{n-1} \oplus \mathbb{C} \cong \beta_{n}^{*} \oplus \beta_{n}
$$

Proof. Using the standard inner product in $\beta_{n-1}$, choose as fibre for $\beta_{n}^{*}$ over $\lambda \in B^{n}$ the orthogonal complement $\lambda^{\perp}$ in $\beta_{n-1} \oplus \mathbb{C}$.

Note that, by taking pull-backs, we have also defined $\beta_{k}^{*}$ over $B^{n}$ for $k=1, \ldots, n-1$ satisfying

$$
\beta_{k-1} \oplus \mathbb{C} \cong \beta_{k}^{*} \oplus \beta_{k}
$$

Iterating, we deduce

$$
\mathbb{C}^{n+1} \cong\left(\bigoplus_{i=1}^{n} \beta_{i}^{*}\right) \oplus \beta_{n}
$$


and substituting successively in (1.1) finally leads to

$$
\tau_{n} \oplus\left(\bigoplus_{i=1}^{n-1}(n-i) \beta_{i}^{*}\right) \oplus \mathbb{R} \cong \mathbb{C}^{\frac{1}{2} n(n+1)} \oplus \mathbb{R} .
$$

This formula allows us to make our first crucial observation.

Proposition 1.5. With the $U$-structure of (1.1), $B^{n}$ is totally normally split.

Proof. From (1.4), we have an isomorphism

$$
v_{n}^{S} \cong \bigoplus_{i=1}^{n-1}(n-i) \beta_{i}^{*}
$$

Using the results of Borel and Hirzebruch [3], we can now compute the integral cohomology ring $H^{*}\left(B^{n}\right)$. Writing $b_{0}, b_{1}, \ldots, b_{n} \in H^{2}\left(B^{n}\right)$ for the respective first Chern classes of $\beta_{0}, \beta_{1}, \ldots, \beta_{n}$ (so that $b_{0}=0$ ), we deduce that $H^{*}\left(B^{n}\right)$ is free over $H^{*}\left(B^{n-1}\right)$ on generators $1, b_{n}$, and that

$$
b_{n}\left(b_{n}-b_{n-1}\right)=0
$$

By induction, this yields the following answer.

Proposition 1.6. (see [4, Section 42]). The ring $H^{*}\left(B^{n}\right)$ is given by

$$
H^{*}\left(B^{n}\right) \cong \mathbb{Z}\left[b_{1}, \ldots, b_{n}\right] /\left(b_{k}^{2}-b_{k} b_{k-1}, 1 \leqq k \leqq n\right)
$$

for all $n>0$.

We observe that we can mimic all the preceding constructions and computations in the symplectic case, beginning with $Q^{0}=*$ and the trivial $S p(1)$ bundle $\chi_{0}=H$. We produce a smooth $4 n$ dimensional manifold $Q^{n}$, carrying $S p(1)$ bundles $\chi_{0}, \ldots, \chi_{n}$. The tangent bundle satisfies

$$
\tau_{n} \oplus \mathbb{R} \cong\left(\bigoplus_{i=0}^{n-1} \chi_{i}\right) \oplus \mathbb{R}
$$

so $\tau_{n}^{S}$ and $v_{n}^{S}$ admit $S p$-structures such that $Q^{n}$ is totally tangentially split. This $S p$ structure may be extended over the corresponding 5-disc bundle.

We then define $S p(1)$ bundles $\chi_{k}^{*}$ over $Q^{n}$ for $k=1, \ldots, n-1$ satisfying

$$
\chi_{k-1} \oplus \mathbb{C} \cong \chi_{k}^{*} \oplus \chi_{k}
$$

and so acquire an isomorphism

$$
v_{n}^{S} \cong \bigoplus_{i=1}^{n-1}(n-i) \chi_{i}^{*}
$$

So $Q^{n}$ is also totally normally split. 
Finally, if we write $q_{0}, q_{1}, \ldots, q_{n} \in H^{4}\left(Q^{n}\right)$ for the respective first symplectic Pontrjagin classes of $\chi_{0}, \chi_{1}, \ldots, \chi_{n}$ (so that $q_{0}=0$ ), we infer the integral cohomology ring structure

$$
H^{*}\left(Q^{n}\right) \cong \mathbb{Z}\left[q_{1}, \ldots, q_{n}\right] /\left(q_{k}^{2}-q_{k-1} q_{k}, 1 \leqq k \leqq n\right) .
$$

\section{Generators for $M U_{*}\left(C P^{\infty}\right)$ and $M S p_{*}\left(H P^{\infty}\right)$}

In this section we utilise Section 1 to introduce a direct approach to creating generators for the reduced bordism modules $M U_{*}\left(C P^{\infty}\right)$ and $M S p_{*}\left(H P^{\infty}\right)$ over the respective coefficient rings. Such a construction does not seem to appear in the literature, although indirect methods have been attempted [5], [7].

Using the notation of $[10]$, we can state the following well-known definitive property.

Proposition 2.1. $M U_{*}\left(C P_{+}^{\infty}\right)$ is a free $M U_{*}$ module on generators $b f_{0}, b f_{1}, b f_{2}, \ldots$, where $b f_{0}=1$, and $b f_{n} \in M U_{2 n}\left(C P^{\infty}\right)$. Furthermore, the $b f_{n}$ 's are uniquely specified $b y$

$$
c f_{1} \cap b f_{n}=b f_{n-1}, \quad n=1,2, \ldots,
$$

where $c f_{1} \in M U^{2}\left(C P_{+}^{\infty}\right)$ is the universal first Conner-Floyd Chern class.

Similarly, $M S p_{*}\left(H P_{+}^{\infty}\right)$ is a free $M S p_{*}$ module on generators $q f_{0}, q f_{1}, q f_{2}, \ldots$, where $q f_{0}=1$, and $q f_{n} \in M S p_{4 n}\left(H P^{\infty}\right)$. Furthermore, the $q f_{n}$ 's are uniquely specified by

$$
p f_{1} \cap q f_{n}=q f_{n-1}, \quad n=1,2, \ldots,
$$

where $p f_{1} \in M S p^{4}\left(H P_{+}^{\infty}\right)$ is the universal first Conner-Floyd Pontrjagin class.

We can now describe our main observation.

Proposition 2.2. The singular manifolds

$$
\beta_{n}: B^{n} \rightarrow C P^{\infty}, \quad \chi_{n}: Q^{n} \rightarrow H P^{\infty}
$$

represent $b f_{n} \in M U_{2 n}\left(C P^{\infty}\right)$ and $q f_{n} \in M S p_{4 n}\left(H P^{\infty}\right)$ respectively.

Proof. We treat the complex case in detail, noting that $\beta_{0}: * \rightarrow C P^{\infty}$ clearly represents 1 .

Since $B^{n}$ bounds for $n>0, \beta_{n}$ represents an element in the reduced bordism module, so by (2.1) it suffices to determine its image under $c f_{1} \cap$. This we can do by making $\beta_{n}$ transverse to $C P^{\infty-1}$.

As required for induction, this yields $\beta_{n-1}: B^{n-1} \rightarrow C P^{\infty}$, where $B^{n-1}$ is identified with the subspace of $B^{n}$ consisting of lines in $\beta_{n-1}$, and as such has $U$-structure equivalent to that given in (1.1).

The symplectic case is entirely analogous.

\section{Generators of $M U_{*}$}

In this section we concentrate on the complex case, and manufacture our set of 
generators for $M U_{*}$. Our detection criterion is the well-documented Milnor genus, e.g. see [14].

First we consider the cartesian product $B^{q} \times B^{r}$, with $q, r=1,2, \ldots$, and the product $U$ structure. We write $\beta_{1, j}, \beta_{2, k}$ respectively for the bundles $\beta_{j} \otimes \mathbb{C}, \mathbb{C} \otimes \beta_{k}$, and hence $b_{1, j}, b_{2, k}$ respectively for their first Chern classes $b_{j} \otimes 1$ and $1 \otimes b_{k}$.

Now dualise the cohomology class $b_{1, q}+b_{2, r}$ to obtain the codimension 2 embedding

$$
B(q, r) \underset{e}{\rightarrow} B^{q} \times B^{r}
$$

where the normal bundles satisfy

$$
v_{q, r} \cong e^{*}\left\{v_{B q \times B^{r}} \oplus\left(\beta_{1, q} \otimes \beta_{2, r}\right)\right\} .
$$

This isomorphism invests $B(q, r)$ with a $U$-structure. It may also, and distinctly, be considered as a projective algebraic variety.

Proposition 3.2. For each $q$ and $r, B(q, r)$ is totally normally and tangentially split.

Proof. Combining (1.5) with (3.1) describes the chosen $U$-structure by

$$
v_{q, r}^{S} \cong e^{*}\left\{\bigoplus_{j=1}^{q-1}(q-j) \beta_{1, j}^{*} \oplus \bigoplus_{k=1}^{r-1}(r-k) \beta_{2, k}^{*} \oplus\left(\beta_{1, q} \otimes \beta_{2, r}\right)\right\}
$$

On the other hand, using (1.3) twice, we obtain

$$
\begin{aligned}
\mathbb{C}^{(q+1)(r+1)} & \cong\left(\bigoplus_{j=1}^{q} \beta_{1, j}^{*} \oplus \beta_{1, q}\right) \otimes\left(\bigoplus_{k=1}^{r} \beta_{2, k}^{*} \oplus \beta_{2, r}\right) \\
& \cong\left(\beta_{1, q} \otimes \beta_{2, r}\right) \oplus \gamma
\end{aligned}
$$

over $B(q, r)$, where

$$
\gamma=\bigoplus_{j, k=1}^{q, r}\left\{\left(\beta_{1, j}^{*} \otimes \beta_{2, r}\right) \oplus\left(\beta_{1, q} \otimes \beta_{2, k}^{*}\right) \oplus\left(\beta_{1, j}^{*} \otimes \beta_{2, k}^{*}\right)\right\}
$$

Thus we can amalgamate (1.1) and (3.1) to describe the same $U$-structure by

$$
\tau_{q, r}^{s} \cong e^{*}\left\{\bigoplus_{j=0}^{q-1} \beta_{1, j} \oplus \bigoplus_{k=0}^{r-1} \beta_{2, k} \oplus \gamma\right\}
$$

as sought.

Corollary 3.3. Any product of the form

$$
B(\mathbf{q}, \mathbf{r})^{\mathrm{m}}=B\left(q_{1}, r_{1}\right)^{m_{1}} \cdots B\left(q_{k}, r_{k}\right)^{m_{k}}
$$

is totally normally and tangentially split. 

by

We may now use the standard methods of [14] to compute the normal Milnor genus

$$
\begin{aligned}
s_{q+r-1}(B(q, r)) & =\left\langle\left(b_{1, q}+b_{2, r}\right)^{q+r}, \mu\right\rangle \\
& =\left(\begin{array}{c}
q+r \\
r
\end{array}\right) \in \mathbb{Z},
\end{aligned}
$$

where $\mu \in H_{2(q+r)}\left(B^{q} \times B^{r}\right)$ is the fundamental class.

This suffices to show that any element $x \in M U_{*}$ may be expressed as an integral linear combination of the form

$$
x=\Sigma x_{\mathbf{q}, \mathbf{r}}^{\mathbf{m}} B(\mathbf{q}, \mathbf{r})^{\mathbf{m}}
$$

In particular, for any prime $p, B(p-1,1)$ represents an algebra generator in $M U_{2 p-2}$, since $s_{p-1}(B(p-1,1))=p$.

To prove our theorem, it remains only to establish three lemmas.

Lemma 3.5. Given two U-manifolds which are totally normally and tangentially split, so is their connected sum.

Proof. Use the fact that $U(n)$ is connected for all $n$.

The symplectic analogue is also true.

Lemma 3.6. The involution $t: B^{n-1} \rightarrow B^{n-1}$ defined by $t(\lambda)=\lambda^{\perp}$ (see (1.2)) satisfies

$$
t^{*} \beta_{n-1}=\beta_{n-1}^{*} \text { and } t^{*} \beta_{k}=\beta_{k} \quad \text { for any } k=0, \ldots, n-2 \text {. }
$$

Proof. Essentially, $t^{*} \beta_{n-1}=\beta_{n-1}^{*}$ by definition. The other equations follow since the diagram

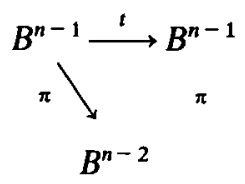

commutes.

Corollary 3.7. The singular manifold

$$
\beta_{n}^{*}: B^{n} \rightarrow C P^{\infty}
$$

represents $-b f_{n} \in M U_{2 n}\left(C P^{\infty}\right)$, for $n>0$.

Proof. We appeal to (2.1) again, noting first that $\beta_{1}^{*}$ clearly represents $-b f_{1}$.

Now suppose that $n>1$. As in (2.2) we apply $c f_{1} \cap$ to the element represented by $\beta_{n}^{*}$. Once more, a simple transversality argument shows that we obtain $\beta_{n-1}^{*}: B^{n-1} \rightarrow C P^{\infty}$, 
where $B^{n-1}$ is identified by the involution $t$ with the subspace of $B^{n}$ consisting of lines in $\beta_{n-1}$.

Hence

$$
\tau_{n-1} \oplus \beta_{n-1}^{*} \cong t^{*} \tau_{n}
$$

so

$$
\begin{aligned}
\tau_{n-1} \oplus \beta_{n-1}^{*} \oplus \mathbb{R} & \cong t^{*}\left(\bigoplus_{i=0}^{n-2} \beta_{i}\right) \oplus \mathbb{R} \\
& =\beta_{n-1}^{*} \oplus \bigoplus_{i=0}^{n-3} \beta_{i} \oplus \mathbb{R}
\end{aligned}
$$

Thus

$$
\tau_{n-1}^{s} \cong \bigoplus_{i=0}^{n-3} \beta_{i}
$$

as sought, and our induction is done.

As before, the symplectic analogue also holds.

Lemma 3.8. For each $q$ and $r$, we can represent the bordism class $-B(q, r)$ by $a$ manifold $A(q, r)$ which is totally normally and tangentially split.

Proof. By (3.7), we can construct $A(q, r)$ by dualising $c_{1}\left(\beta_{1, q}^{*} \otimes \beta_{2, r}\right)$ in $H^{2}\left(B^{q} \times B^{r}\right)$, where

$$
c_{1}\left(\beta_{1, q}^{*} \otimes \beta_{2, r}\right)=b_{1, q-1}-b_{1, q}+b_{2, r}
$$

Following (3.2), this yields

$$
A(q, r) \underset{e}{\longrightarrow} B^{q} \times B^{r}
$$

whose $U$-structure is given by

$$
v_{q, r}^{s} \cong e^{*}\left\{\bigoplus_{j=1}^{q-1}(q-j) \beta_{1, j}^{*} \oplus \bigoplus_{k=1}^{r-1}(r-k) \beta_{2, k}^{*} \oplus\left(\beta_{1, q}^{*} \otimes \beta_{2, r}\right)\right\}
$$

and

$$
\tau_{q, r}^{s} \cong e^{*}\left\{\bigoplus_{j=0}^{q-1} \beta_{1, j} \oplus \bigoplus_{k=0}^{r-1} \beta_{2, k} \oplus \gamma\right\}
$$

where

$$
\gamma=\left(\beta_{1, q} \otimes \beta_{2, r}\right) \oplus \bigoplus_{j, k=1}^{q-1, r}\left\{\left(\beta_{1, j}^{*} \otimes \beta_{2, r}\right) \oplus\left(\beta_{1, q-1} \otimes \beta_{2, k}^{*}\right) \oplus\left(\beta_{1, j}^{*} \otimes \beta_{2, k}^{*}\right)\right\}
$$

These results may now be combined. 
Theorem 3.9. Any element of $M U_{*}$ may be represented by a connected $U$-manifold which is totally normally and tangentially split.

Proof. Let the element of $M U_{*}$ be $x$, and expand it as in (3.4).

For each positive integer $x_{\mathbf{q}, r}^{\mathrm{m}}$, the corresponding term $x_{\mathbf{q}, \mathbf{r}}^{\mathrm{m}} B(\mathbf{q}, \mathbf{r})^{\mathrm{m}}$ is suitably represented by utilising (3.3) and (3.5). On the other hand, if $x_{q, \mathrm{r}}^{\mathrm{m}}$ is negative we use (3.8) and (3.5). Final appeal to (3.5) then allows us to bring all terms together.

\section{Representing $\phi$ 's in $M S p_{*}$}

In this section we turn our attention to some implications of the $S p$ version of (2.2).

First we recall some results of [9].

Let $\eta$ denote the real Hopf line bundle over $S^{\mathbf{1}}$, and $\xi$ its symplectic counterpart over $H P^{\infty}$ (or any $H P^{n}$ ). Thus $\eta \otimes_{\mathrm{R}} \xi$ is also an $S p(1)$ bundle over $S^{1} \times H P^{\infty}$, and as such it has a first Conner-Floyd Pontrjagin class

$$
p f_{1}\left(\eta \otimes_{\mathrm{R}} \xi\right) \in M S p^{4}\left(S^{1} \times H P^{\infty}\right)
$$

But

$$
\operatorname{MSp}^{*}\left(S^{1} \times H P_{+}^{\dot{\infty}}\right) \cong \operatorname{MSp}^{*}\left[\left[p f_{1}, h\right]\right] /\left(h^{2}\right)
$$

so there is an expansion of the form

$$
p f_{1}\left(\eta \otimes_{\mathrm{R}} \xi\right)=p f_{1}+\sum_{n \geqq 0} h \theta_{n} p f_{1}^{n}
$$

where $\theta_{n} \in M S p_{4 n-3}, n=1,2, \ldots$ In fact $\theta_{1}$ is the generator of $M S p_{1} \cong \mathbb{Z} / 2$, represented by $S^{1}$ with the twisted framing, and $\theta_{2 m+1}=0$ for $m>0$.

In contrast, the elements $\theta_{2 m}$, suitably relabelled $\phi_{m}$, are non-zero and indecomposable elements of order 2 in $M S p_{*}$. By all accounts, (see [6], [17]), they play a fundamental role in $M S p_{*}$, whose overall structure remains, of course, a mystery.

We now show how to give precise representatives for the elements $\phi_{n}$ using Section 2 . This has been attempted before in [1] and [11], but with success only modulo certain decomposables.

Note first from (4.1) that

$$
\phi_{n}=\left\langle p f_{1}(\eta \otimes \mathbb{R} \xi), g \otimes q f_{2 n}\right\rangle
$$

where $g \in M S P_{1}\left(S^{1}\right)$ is the dual generator to $h$, and represented by the identity on $S^{1}$. But employing (2.2) with $n>0, g \otimes q f_{2 n}$ is represented by $t: R^{8 n+1} \rightarrow H P^{2 n}$, where $R^{8 n+1}=S^{1} \times Q^{2 n}$ and $t$ classifies $\eta \otimes_{\mathbf{R}} \chi_{2 n}$. So $\phi_{n}$ arises from making $t$ transverse to $H P^{2 n-1}$.

Proposition 4.2. The element $\phi_{n}$ may be represented on $R^{8 n-3}$ by an Sp structure which is totally normally and tangentially split. 
Proof. The transversality described above yields

$$
R^{8 n-3}=S^{1} \times Q^{2 n-1} \stackrel{j}{\longrightarrow} S^{1} \times Q^{2 n}
$$

where $j$ embeds $Q^{2 n-1}$ in $Q^{2 n}$ as the subspace of quaternionic lines in $\chi_{2 n-1}$. The corresponding normal $S p$ structure on $S^{1} \times Q^{2 n-1}$ is provided by the isomorphism

$$
v_{R^{8 n-3}} \cong v(j) \oplus j^{*} v_{R^{8 n+1}}
$$

Moreover, $v(j) \cong \eta \otimes_{\mathrm{R}} \chi_{2 n-1}$ by construction, whilst from (1.8)

$$
v_{R^{8 n+1}} \cong \mathbb{R} \otimes_{\mathrm{R}}\left(\bigoplus_{k=1}^{2 n-1}(2 n-k) \chi_{k}^{*}\right)
$$

Thus

$$
v_{R}{ }^{8 n-3} \cong\left(\eta \otimes_{R} \chi_{2 n-1}\right) \oplus\left(\mathbb{R} \otimes_{R}\left(\bigoplus_{k=1}^{2 n-1}(2 n-k) \chi_{k}^{*}\right)\right)
$$

and $R^{8 n-3}$ is totally normally split.

Similarly, recalling (1.7) and the fact that $2 \eta \cong \mathbb{R}^{2}$ gives

$$
\tau_{R^{8 n-3}} \cong\left(\eta \otimes_{\mathrm{R}} \chi_{2 n-1}\right) \oplus\left(\mathbb{R} \otimes_{\mathrm{R}}\left(\bigoplus_{k=1}^{2 n-1} \chi_{i}\right)\right),
$$

completing the proof.

In fact a simply-connected representative for $\phi_{n}$ may also be given by these methods, by choosing a suitable $S p$ structure on the $S^{5}$ bundle $S\left(\chi_{2 n-2} \oplus \mathbb{R}^{2}\right)$ over $Q^{2 n-2}$.

In conclusion, we remark that the procedures introduced in this paper appear to have a wider applicability to $M S p_{*}$. Most of the constructions of $S p$ manifolds given in [12] and [15] can be adapted to give results which are totally normally and tangentially split. This suggests that the infinite product $\bigwedge_{\infty} H P^{\infty}$ may have $M S p$ as a wedge summand, thus mimicking the complex case.

\section{REFERENCES}

1. J. C. Alexander, A family of indecomposable symplectic manifolds, Amer. J. Math. 94 (1972), 699-710.

2. R.Arthan and S. Bullett, The homology of $M O(1)^{\wedge}$, and $M U(1)^{\wedge} \infty, J$. Pure Appl. Algebra 26 (1982), 229-234.

3. A. Borel and F. Hirzebruch, On characteristic classes of homogeneous spaces: I, Amer. J. Math. 80 (1958), 458-538.

4. P. E. Conner and E. E. Floyd, Differentiable Periodic Maps (Springer, 1964). 
5. P. E. Conner and E. E. Floyd, Torsion in SU-bordism, Mem. Amer. Math. Soc. 60 (1966).

6. S. O. Kochman, The symplectic cobordism ring: I, Mem. Amer. Math. Soc. 228 (1980).

7. P. S. Landweber, On the symplectic bordism groups of the spaces $S p(n), H P(n)$ and $B S p(n)$, Michigan Math. J. 15 (1968), 145-153.

8. S. Ochanine and L. Schwartz, Une remarque sur les générateurs du cobordism complexe, Math. Z., to appear.

9. N. RAY, Indecomposables in Tors $M S p_{*}$, Topology 10 (1971), 261-270.

10. N. RaY, Some results in generalised homology, $K$-theory and bordism, Proc. Cambridge Philos. Soc. 71 (1972), 283-300.

11. N. RAY, R. SwItzer and L. TAYLOR, Normal structures and bordism theory, with applications to $M S p_{*}$, Mem. Amer. Math. Soc. 193 (1977).

12. N. Ray, Realizing symplectic bordism classes, Proc. Cambridge Philos. Soc. 71 (1972), 301305 .

13. R. E. Stong, private communication.

14. R. E. Stong, Notes on Cobordism Theory (Princeton Univ. Press, 1968).

15. R. E. Stong, Some remarks on symplectic cobordism, Ann. of Math. 86 (1967), 425-443.

16. R. H. Szczarba, On the tangent bundles of fibre spaces and quotient spaces, Amer. $J$. Math. 86 (1964), 685-697.

17. V. V. VERSHININ, Symplectic cobordism with singularities, Math. USSR-Izv. 22 (1984), $211-$ 226.

Department of Mathematics

THE UNIVERSITY

MANCHESTER

M13 9PL 